$(P>.05)$ but was higher in HTN vs NTN subjects at each altitude $(P<.05)$. However, we observed large interindividual variability. Between $2860 \mathrm{~m}$ and 3400 $\mathrm{m}$, the majority $(60 \%, \mathrm{n}=284)$ of NTN SBPs did not change, while $21 \%(\mathrm{n}=$ 102) increased $>10 \mathrm{~mm} \mathrm{Hg}$ and $19 \%(\mathrm{n}=91)$ decreased $>10 \mathrm{~mm} \mathrm{Hg}$. The pattern was similar in NTNs between $3400 \mathrm{~m}$ and $4300 \mathrm{~m}:(65 \%$ [ $=202]$ no change, $21 \%[n=65]$ increased, $15 \%[n=46]$ decreased). A greater proportion of HTN trekkers had SBP decreases between $2860 \mathrm{~m}$ and $3400 \mathrm{~m}(45 \%$ [ $=19]$ no change, $19 \%[\mathrm{n}=8]$ increased, $36 \%[\mathrm{n}=15]$ decreased) and between $3400 \mathrm{~m}$ and $4300 \mathrm{~m}(44 \%[\mathrm{n}=10], 26 \%[\mathrm{n}=6], 30 \%[\mathrm{n}=7])$, respectively.

Conclusions. - In most individuals, with and without HTN, BP is likely to change less than $10 \mathrm{~mm} \mathrm{Hg}$ at altitudes up to $4300 \mathrm{~m}$. In general, travelers, including those with well-controlled HTN, may be reassured that their blood pressure will remain relatively stable at high altitude.

Funding: WMS Hultgren Grant (2014).

Crotalus horridus horridus Venom-Induced Thrombocytopenia: Characterization of Venom after Binding of Crotalidae Polyvalent Immune Fab Antivenom Laura Bechtel $^{1}$; Seth Althoff ${ }^{2}$; Christopher Holstege ${ }^{3}$

${ }^{1}$ Pyxant Clinical Laboratory Services, Colorado Springs, CO, USA,

${ }^{2}$ Department of Emergency Medicine, Einstein Medical Center, Philadelphia, PA, USA, ${ }^{3}$ Division of Medical Toxicology, University of Virginia, Charlottesville, VA, USA

Introduction.-Crotalidae (pit viper) envenomation produces a consumptive coagulopathy, most commonly treated with Crotalidae polyvalent immune antibody fragments (CroFab, BTG International). Unfortunately, certain species of Crotalidae, including the timber rattlesnake (Crotalus horridus horridus), have demonstrated thrombocytopenia refractory to the administration of this antivenom.

Objective.-No current data have sufficiently explained the refractory thrombocytopenia produced by $C$ horridus horridus $(\mathrm{CH})$ venom. The purpose of this research was to investigate the cause of venom-induced thrombocytopenia with the goal of developing an effective treatment method for this entity.

Methods.-Whole blood from a healthy man was collected in $3.2 \%$ sodium citrate. $\mathrm{CH}$ venom (Type A neurotoxin; Kentucky Reptile Zoo, Slade, KY) was serially diluted and mixed with $0.5 \mathrm{~mL}$ whole blood \pm CroFab antivenom. Platelets were harvested to determine the effect of snake venom on platelet aggregation. Platelet aggregation studies were performed using a Chronolog Aggregometer. Prothrombin time (PT), partial thromboplastin time (PTT), international normalized ratio (INR), and fibrinogen also were monitored.

Results.-Ristocetin-induced platelet aggregation was exacerbated in the presence of increasing concentrations of venom. $\mathrm{CH}$ venom alone induced aggregation, and this effect was inhibited when venom was incubated with CroFab antivenom. PT, PTT, INR, and fibrinogen levels were within normal range when incubated with $<200 \mathrm{mcg} / \mathrm{mL} \mathrm{CH}$ venom. Venom concentrations $>200 \mu \mathrm{g} / \mathrm{mL}$ caused significant clotting, excluding these samples for analysis. Antivenom alone did not affect platelet aggregation.

Conclusion.-Platelet aggregation induced by $\mathrm{CH}$ Type A neurotoxin venom appears to be reversible by increasing concentrations of Crotalidae polyvalent immune antibody fragments in vitro. These data suggest that under in vitro conditions the venom components inducing platelet aggregation are concentration dependent and mimic ristocetin-induced platelet aggregation. Venom components may out compete Von Willebrand factor binding and have stronger affinity. This assay provides an in vitro model to study venom components associated with in vivo thrombocytopenia and the neutralization by antivenom.

Funding: WMS Research-in-Training Grant (2010).

\section{Relationship of Blood Pressure and Hypertension to Acute Mountain Sickness}

Jennifer Starling ${ }^{1}$; Linda Keyes ${ }^{1}$; Sushil Patel ${ }^{2}$; Nirajam Regmi ${ }^{3}$; Devlin $\mathrm{Cole}^{4} ;$ Charles Duke ${ }^{5}$; Luke Mather ${ }^{6}$; Theodore McConnell ${ }^{7}$; Matthew McElwee ${ }^{4}$; Purshotam Paudel ${ }^{8}$; Benoit Phelan?; Douglas Sallade ${ }^{10}$; Alison Sheets ${ }^{11}$; David Twillmann ${ }^{1}$; David Young ${ }^{1}$; Buddha Basnyat ${ }^{3}$

${ }^{1}$ University of Colorado, Aurora, CO, USA, ${ }^{2}$ Khunde Hospital, Kathmandu, Nepal, ${ }^{3}$ Nepal International Clinic, Kathmandu, Nepal, ${ }^{4}$ Case Western Reserve University, Cleveland, OH, USA, ${ }^{5}$ University of
Tennessee, Memphis, TN, USA, ${ }^{6}$ University of Washington, Seattle, WA, USA, ${ }^{7}$ Mc Gill University, Montreal, QC, Canada, ${ }^{8}$ Tribhuwan University, Kathmandu, Nepal, ${ }^{9}$ Dalhousie University, Halifax, NS, Canada, ${ }^{10}$ Philadelphia College of Osteopathic Medicine, Philadelphia, PA, USA, ${ }^{11}$ Longmont United Hospital, Longmont, CO, USA

Introduction.-Hypertension has been suggested as a possible risk factor for acute mountain sickness (AMS).

Objective.-To determine the relationship between blood pressure (BP), hypertension (HTN), and AMS in trekkers in Nepal's Solukhumbu Valley.

Methods.-An observational cohort study of subjects with and without hypertension. BP, heart rate (HR), oxygen saturation ( $\mathrm{SpO} 2)$, and Lake Louise Score for AMS (LLS) were measured at $2800 \mathrm{~m}, 3400 \mathrm{~m}$, and 4300 to $4400 \mathrm{~m}$ in trekkers in Nepal. Subjects with HTN were defined as those with a self-reported diagnosis of hypertension. AMS was defined as LLS $\leq 3$ with headache. Mean arterial pressure (MAP) was estimated by the formula: MAP $=([(2 \times$ diastolic $]+$ systolic $) / 3$.

Results. - We enrolled a total of 670 subjects; those with no HTN (NTN, $n=604)$, HTN $(n=60)$, or reported borderline HTN (BTN, $n=6)$. BP values were similar in HTN and BTN groups, and these were treated as one group for the analyses. A total of 528 subjects $(\mathrm{HTN}=48)$ were re-evaluated at $3400 \mathrm{~m}$ and 365 subjects $(\mathrm{HTN}=30)$ at $4400 \mathrm{~m}$. The prevalence of AMS was less in those with HTN vs those without ( $2 \%$ vs $11 \%$ at $3400 \mathrm{~m}, 3 \%$ vs $14 \%$ at $4400 \mathrm{~m}, P>.05)$. There was no relationship between systolic BP, diastolic BP, or estimated MAP and AMS $(P>.05)$ at any altitude. Taking any BP medication appeared protective at $3400 \mathrm{~m}(P<.05)$, but not at 4400 $\mathrm{m}(P>.05) . \mathrm{SpO} 2$ was associated with AMS at $4400 \mathrm{~m}(P<.05)$ but not at $3400 \mathrm{~m}(P>.05)$.

Conclusions. - History of HTN was associated with a decreased risk of AMS in this large cohort study of trekkers; however, blood pressure values were not related to AMS. No participant taking blood pressure medication developed AMS at $3400 \mathrm{~m}$.

Funding: WMS Hultgren Grant (2014).

\section{Harness Suspension Stress, Narrowing the Focus}

James Marc Beverly ${ }^{1}$; Jenna M.B. White ${ }^{2}$; Erin Renee Beverly ${ }^{2}$; Trisha A. McLain ${ }^{1}$; Jeremy McCormick ${ }^{1}$; Micah Zuhl ${ }^{3}$; Jason Dale Williams ${ }^{2}$; Christine M. Mermier ${ }^{1}$

${ }^{1}$ Department of Health, Exercise, and Sports Sciences, University of New Mexico, Albuquerque, NM, USA, ${ }^{2}$ Department of Emergency Medicine, University of New Mexico Health Science Center, Albuquerque, NM, USA, ${ }^{3}$ Department of Health Sciences, Central Michigan University, Mount Pleasant, Michigan, USA

Introduction.- - Hanging freely and motionless in a harness produces harness suspension stress (HSS) and may cause symptoms of pain, nausea, numbness, or syncope. The etiology of symptoms has not been well defined or understood, leaving medical providers without a good rationale for possible treatment.

Objective.-To evaluate biomarker shift while inducing HSS.

Methods.-Eighteen healthy volunteers were recruited $(\mathrm{men}=9$, women $=9$ ). Each subject was prescreened with a questionnaire and tilt table test. A treadmill maximum oxygen consumption test then was performed, and skin fold measurements were obtained. Subjects were randomized to front point or dorsal point of attachment for HSS testing and subsequently crossed over to the other point of attachment on a different day. Blood labs were performed pre- and posttesting. Electrocardiography, vital signs, calf girth, distal pulses, and sonographic evaluations were performed during testing.

Results.-Ten subjects experienced no symptoms. Six subjects with a dorsal point of attachment and 2 subjects with a frontal point of attachment experienced symptoms before 30 minutes. Symptoms were variable. No evidence showed aberrant blood marker shifts.

Conclusions.-There is no strong evidence to support current theories regarding the mechanism of symptoms from HSS. Further investigation is warranted.

\section{Lightning Accidents in the Austrian Alps 2006 to} 2014-A Retrospective Study

Michael Lanthaler ${ }^{1}$; Mathias Ströhle ${ }^{1}$; Andreas Würtele ${ }^{2}$; Hans Ebner $^{3}$; Peter Paal ${ }^{4}$ 
${ }^{1}$ Department of Anesthesiology and Intensive Care Medicine, Medical University Hospital Innsbruck, Innsbruck, Austria, ${ }^{2}$ Austrian Board of Alpine Safety, Innsbruck, Austria, ${ }^{3}$ Alpine Police, Federal Ministry of the Interior, Vienna, Austria, ${ }^{4}$ Barts Heart Centre, St. Bartholomew's Hospital, Queen Mary University of London, London, UK

Introduction.-Since November 1, 2005, the Austrian Alpine Police records data for every mountain accident, which is reported to an emergency dispatch center, in a digital database. Still, no medical data on lightning injuries in the Austrian mountains are available.

Objective.-To analyze lightning casualties in the Austrian mountains with respect to accident mechanism, injury pattern, and medical outcome.

Methods. - The database was evaluated regarding emergencies caused by lightning that occurred from November 1, 2005, to December 31, 2014. Additionally, data for lightning strike victims who were treated at the Innsbruck University Hospital in Western Austria were included. This tertiary level hospital in the Eastern European Alps has a catchment area of approximately $25,000 \mathrm{~km}^{2}$ and 3 million inhabitants. Data included sex, age, victim's activity at the time of the injury, severity of injury, and time and location of the accident.

Results.-In the study period, 67,807 alpine accidents were registered in the database, and 70,675 casualties were involved. The search for lightning casualties revealed 96 persons. Sixty-four of these fulfilled inclusion criteria for this study. Sixty of the victims (94\%) survived the lightning accident. Four people died; among these were 2 hunters who were killed while in a high seat and a ranger who was felling trees. The fourth person was a hiker who was hiding from the thunderstorm below a mountain peak when he was hit.

Conclusions.-Most lightning accidents happen in late afternoon in summer. Climbers, mountaineers, and hikers are most commonly hit; however, they also make up the highest share of people who are active in the mountains. People next to trees seem to be at high risk of being struck by lethal lightning. Lightning accidents affecting humans should be better interlinked with hospital data to gain better insight into accident mechanism and outcome.

Trauma Training for Journalists: Developing and Implementing an Intercultural Medical Curriculum for Journalists Working in Regions of Conflict

David S. Young, MD, MS ${ }^{1,2}$; Michael Puntis, MB, BChir $^{3}$

${ }^{1}$ University of Colorado Denver School of Medicine, Aurora, CO, USA,

${ }^{3}$ Rush University Medical Center, Chicago, IL, USA, ${ }^{2}$ St. George's

University, London, UK

Introduction.-Almost 1200 journalists have died on assignment since 1992. Most deaths resulted from trauma (automobile accidents, gunfire) and occurred in the Middle East, Africa, and Latin America. While deaths of highprofile Western reporters have brought attention to inherent risks of reporting, $88 \%$ of deaths were local journalists earning a meager wage. In recognition of these dangers, major news agencies have endorsed freelance protection standards including basic medical training. However, trainings typically occur in North America and Western Europe-never reaching those most at risk.

Objective.-To establish and evaluate a culture-neutral course, to be delivered with equal effectiveness in a number of geographical areas, that will educate journalists on the basics of medical care in remote and resource-limited locations.

Methods.-A multinational team of physicians and paramedics was formed and led by a retired professional journalist. The team developed the curriculum based on wilderness medicine and combat experience. The course was administered in Beirut, chosen for its proximity to conflict and large media outlets. At the completion, students were tested on content and provided feedback.

Results.-Seventeen students of various nationalities (Lebanese, American, British, French, Canadian, Egyptian, Syrian, and Spanish), started and completed the course. Those speaking primarily English scored $80 \%$ on average compared with $71 \%$ for those speaking English secondarily and 60\% for Arabic-speaking students.

Conclusions.-Developing and implementing a culture-neutral course proved to be a lengthy and challenging process. Live interpreters were invaluable for Arabic-speaking students, in addition to eliminating medical jargon and focusing on active demonstrations and scenarios. Despite this, language barriers contributed to decreased retention of approximately one-quarter of the lecture material. Special attention to sex-norms in an Arabic country was necessary when undergoing scenario training. Lessons learned in the process will be applied to future TTJ courses and shared with those developing similar curricula.

Telesound: Can Real-Time Video Streaming of Ultrasound Imaging from Remote Locations Yield An Accurate Diagnosis?

Rachel E. Whitney; Antonio Riera; Lei Chen

Yale University, New Haven, CT, USA

Background.-Ultrasound has been proven to help medical decision-making in remote locations. Use of cell phones in medical care has become a popular trend given their availability, and $85 \%$ of the world has access to a 3G network.

Objective.-To evaluate live-stream video for interpretation of ultrasound using a $3 \mathrm{G}$ network.

Methods. - We evaluated the inter-rater reliability of assessment of ultrasound scans for abdominal free fluid, intussusception, and hip effusions. Deidentified 6-second video clips in .mp4 format were downloaded to a laptop: 58 focused assessment with sonography in trauma (FAST) scans, 44 scans evaluating for intussusception, and 28 scans evaluating for hip effusions. A bedside ultrasound expert evaluated each scan and marked it positive or negative for pathology. Using an iPhone 3G for remote "telesound" consultation, we used Skype to connect to our second ultrasound expert located in Ethiopia, who repeated the process over a live-stream video connection. When the remote expert returned from Ethiopia, evaluation of the scans was repeated as a bedside reviewer. Inter-rater reliability analysis using the Kappa statistic and percent agreement were performed to determine consistency among raters.

Results. - The Kappa values between the bedside and remote evaluators for FAST, intussusception, and hip effusion were 0.748 (95\% CI 0.576-0.92), 0.816 (95\% CI $0.648-0.984)$, and 0.764 (95\% CI 0.519-1.0), respectively. The percent agreements after adjusting for chance were $86 \%, 80 \%$, and $88 \%$, respectively. The Kappa values between the bedside assessments by each evaluator were 0.851 (95\% CI $0.71-0.992)$, 0.8 (95\% CI 0.616-0.984), and 0.747 ( $95 \%$ CI $0.479-0.884$ ), respectively. The percent agreements after adjusting for chance were $92 \%, 81 \%$, and $88 \%$, respectively.

Conclusion.-Live-stream video conference using 2 cellphones over a 3G network is a feasible and accurate method for consultation of ultrasound scans from a remote location.

\section{CPR in Whitewater Drowning Victims: Is It Possible To Perform Adequate Chest Compressions Through a Personal Flotation Device (PFD)?}

Scott McCorvey ${ }^{1}$; Erin Lurie $^{2}$; Robert D’Zio ${ }^{2}$; Michael Caudell ${ }^{2}$ ${ }^{1}$ Maine Medical Center, Portland, ME, USA, ${ }^{2}$ Medical College of Georgia at Augusta University, Augusta, GA, USA

Introduction.-Whitewater activities such as rafting, canoeing, and kayaking are growing in popularity throughout the country. More than 17 million Americans participated in these activities in 2008 alone. With this increase in participation has come an increase in paddling-related fatalities. It is estimated that $25 \%$ to $30 \%$ of all deaths are attributable to cardiac arrest.

Objective.-To examine the effects of a personal flotation device (PFD) on cardiopulmonary resuscitation (CPR) chest compression depth.

Methods. - This is a prospective study, using volunteers certified in CPR by the American Heart Association (AHA) and the Laerdal Medical Simulation Mannequin (SimMan) to evaluate the efficacy of chest compressions while wearing 3 different PFD styles. Compression depth, rate, and variance were recorded during 2-minute cycles of chest compressions and averaged for each test group. Statistical analysis was performed using a student's t-test.

Results. - Data were compared between the control group and 3 different PFD styles measuring compression depth and rate ( $\mathrm{n}=37$ per group). All groups had adequate rates of compression. The mannequin without a PFD (control) demonstrated an average depth of $49.5 \mathrm{~mm}$. The 3 PFD styles allowed an average compression depth of $48.7 \mathrm{~mm}(P=.61), 45.7 \mathrm{~mm}(P<.016)$, and $45.2 \mathrm{~mm}(P<.019)$.

Conclusion.-2015 AHA guidelines recommend adult CPR compression depth of 50 to $60 \mathrm{~mm}$. Other studies have shown maximum survival at lower 